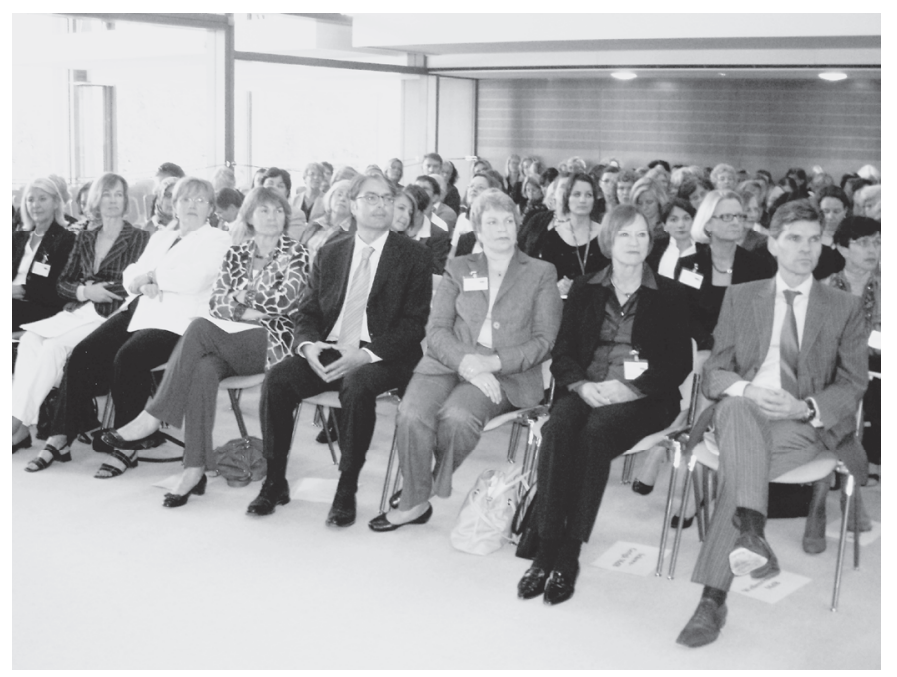

$\Delta \quad$ In der ersten Reihe (v.r.n.I.): Ingo Wellenreuther MdB, Irmingard ScheweGerigk MdB, Sibylle Laurischk MdB, Johannes Jung MdB, Präsidentin des BAG Ingrid Schmidt, Richterin am EGMR Dr. h.c. Renate Jaeger, Generalanwältin am EuGH Prof. Dr. Dr. Juliane Kokott, Präsidentin des Landesverfassungsgerichtshofs Berlin Margret Diwell (Foto: $C D$ )

Meine Damen und Herren,

Integration funktioniert besonders gut durch den Beruf, also am Arbeitsplatz. Aus diesem Grund ist auch die Anerkennung ausländischer Bildungsabschlüsse ein ganz wichtiger Aspekt der Integration. Viele Zuwanderer sind in ihrer Heimat gut ausgebildet worden und müssen dann hier erleben, dass ihre Qualifikationen nicht anerkannt werden. Damit lassen wir nicht nur ein enormes Potential ungenutzt, sondern wir sorgen auch für Frust bei den Zuwanderern und erschweren die Integration. Wir brauchen deshalb eine bessere Anerkennung der Abschlüsse und eine solide rechtliche Grundlage hierfür. Wir brauchen auch mehr Teilhabe für diejenigen, die keinen deutschen Pass haben. Warum lassen wir zwar EU-Bürgerinnen in den Kommunen wählen, aber nicht die rund vier Millionen Menschen aus der Türkei, Serbien oder etwa Kroatien, die dauerhaft bei uns leben? Dies sind nur drei Beispiele dafür, wie gesetzliche Regelungen helfen können, dass Menschen unterschiedlicher Herkunft glücklich und friedlich zusammenleben. Ich bin mir sicher, dass der djb und dieser Kongress noch weitere Anregungen geben, wie wir das erreichen können, und deshalb bin ich auf Ihre rechtspolitischen Empfehlungen in dieser Sache auch sehr gespannt.

Meine Damen und Herren,

lassen sie mich noch ein Wort sagen zu einem ganz anderen Thema, das dem djb und mir ebenfalls sehr am Herzen liegt: Es geht um Frauen in den Aufsichtsräten und in den Vorständen deutscher Unternehmen. In der vergangenen Woche hat hier in dieser Stadt das Karlsruhe Institute of Technology eine bemerkenswerte Studie vorgestellt. Sie untersucht erstmals die 600 führenden Unternehmen in Deutschland. Die Zahlen, die dabei herausgekommen sind, sind frustrierend: Von 1721 Vorständen sind ganze 42 Frauen. Das sind 2,4 Prozent. Bei den Aufsichtsräten sind es gut acht Prozent. Nennenswerte positive Veränderungen hat es in den letzten zehn Jahren nicht gegeben. Im Gegenteil, seit 2005 ist der Frauenanteil sogar wieder leicht rückläufig. Der Deutsche Juristinnenbund und auch ich setzen uns seit geraumer Zeit dafür ein, dass endlich Schluss ist mit der reinen Männerwirtschaft in den Führungsetagen deutscher Unternehmen. Angesichts dieser neuen Zahlen wird für mich aber eines sehr deutlich: Appelle, Förderprogramme und guter Wille allein reichen nicht aus. Ganz offenkundig ist auch hier eine „Integration durch Recht“ erforderlich. Ich meine, es war deshalb richtig, was meine Partei in ihrem Regierungsprogramm festgeschrieben hat: Wir wollen nach dem Vorbild anderer Länder eine Frauenquote für Aufsichtsräte schaffen. Dieses Ziel zu erreichen, wird gewiss nicht einfach weder politisch noch juristisch. Trotzdem werden wir es politisch angehen - Jutta Wagner hat recht: Die Zeit ist reif dafür.

Bei diesem und vielen anderen Vorhaben biete ich dem Deutschen Juristinnenbund auch künftig eine enge Zusammenarbeit an. Der djb ist und bleibt für das Bundesjustizministerium ein ganz wichtiger Partner. Deshalb wünsche ich Ihnen weiterhin eine erfolgreiche Arbeit und einen erfolgreichen Kongress hier in Karlsruhe.

\title{
Grundrechte ziehen klare Grenzen und eröffnen Räume der Teilhabe
}

\author{
Prof. Dr. Ulrich Goll MdL (FDP) \\ Justizminister und Integrationsbeauftragter der Landesregie- \\ rung Baden-Württemberg, Stuttgart
}

Sehr geehrte Frau Präsidentin Wagner, sehr geehrter Herr Bundesverfassungsgerichtspräsident Professor Papier, sehr geehrte
Frau Kollegin Zypries, sehr geehrte Frau Professor LübbeWolff, sehr geehrte Damen und Herren,

im Namen der Landesregierung und auch persönlich begrüße ich Sie alle hier in Karlsruhe sehr herzlich zum 38. Kongress des Deutschen Juristinnenbundes. Angesichts der Tatsache, dass von den rund 15 Millionen in Deutschland lebenden 
Menschen mit Migrationshintergrund fast die Hälfte Mädchen und Frauen sind, begrüße ich es sehr, dass Sie für den diesjährigen Kongress das Thema „Integration durch Recht Frauen mit Migrationshintergrund zwischen Teilhabe und Ausgrenzung “ ausgewählt haben.

Die Bundesrepublik Deutschland ist ein Raum der Freiheit und des Rechts. „Integration durch Recht“ bedeutet für mich daher zuallererst, dass wir uns als Einwanderungsgesellschaft über die Kultur des Zusammenlebens in unserem Land einig sein müssen: Dabei sind Demokratie, Rechtsstaatlichkeit, die Grund- und Menschenrechte, die Achtung gegenüber Andersdenkenden, Andersgläubigen oder Nichtgläubigen sowie die Gleichberechtigung der Geschlechter die für alle geltenden unumstößlichen Fundamente, die universellen Werte unserer Gesellschaft, über die sich niemand hinwegsetzen darf und die auch nicht verhandelbar sind. Abweichende kulturelle, religiöse oder traditionelle Überzeugungen können keine Rechtfertigung dafür sein, diese elementaren Regeln in Frage zu stellen oder zu missachten.

Diese Grundlagen und Grundrechte ziehen klare Grenzen, eröffnen aber auch Räume der Teilhabe und der persönlichen Entfaltung. Dies gilt insbesondere für Mädchen und Frauen mit Migrationshintergrund. Migrantinnen, Ausländerinnen, Aussiedlerinnen, Zuwanderinnen, Frauen nichtdeutscher Herkunft, Frauen mit Migrationshintergrund: Es gibt viele Bezeichnungen, häufig jedoch noch zu wenig gesicherte Daten und Fakten über den Alltag von Mädchen und Frauen, die nach Deutschland gekommen sind oder als Kinder von $\mathrm{Zu}$ wanderern hier geboren wurden, aufwachsen, arbeiten, Familien gründen und alt werden. Denn Statistiken unterscheiden vielfach nur zwischen Deutschen und Ausländern beziehungsweise Migranten, ohne aber nach Geschlechtern zu differenzieren. Ein Bericht des UN-Sekretariats von 1995 nannte Migrantinnen daher sogar „die unsichtbare Hälfte“. Erst seit wenigen Jahren rücken in Politik, Wissenschaft und Öffentlichkeit auch Migrantinnen verstärkt ins Blickfeld. Die speziellen Lebenslagen und Bedürfnisse von Frauen und Mädchen mit Migrationshintergrund gewinnen immer mehr an Bedeutung und Aufmerksamkeit, so etwa im Nationalen Integrationsplan und auch auf diesem Kongress.

Die meisten Mädchen und Frauen mit Migrationshintergrund sind gut integriert und keineswegs, wie manche Medien gerne berichten, zurückgeblieben in einer unentrinnbaren $\mathrm{Op}$ ferrolle. Die vorliegenden Erkenntnisse zeigen, dass die Lebensweisen und Lebenswelten der Migrantinnen weder integrationshemmend noch emanzipationshinderlich sind - im Gegenteil. Viele - gerade Frauen und Mädchen aus der zweiten und dritten Generation - sind überwiegend bildungs- und familienorientiert, streben finanzielle Unabhängigkeit sowie partnerschaftliche Gleichberechtigung an. Starke familiäre Bindungen bedeuten für sie in der Regel keine Einengung, sondern eine wesentliche Ressource. Mädchen mit Migrationhintergrund sind in den höherwertigen Bildungsgängen gegenüber den Jungen überrepräsentiert. Bei den Schulabschlüssen ergibt sich das gleiche Bild: Mädchen ausländischer Herkunft

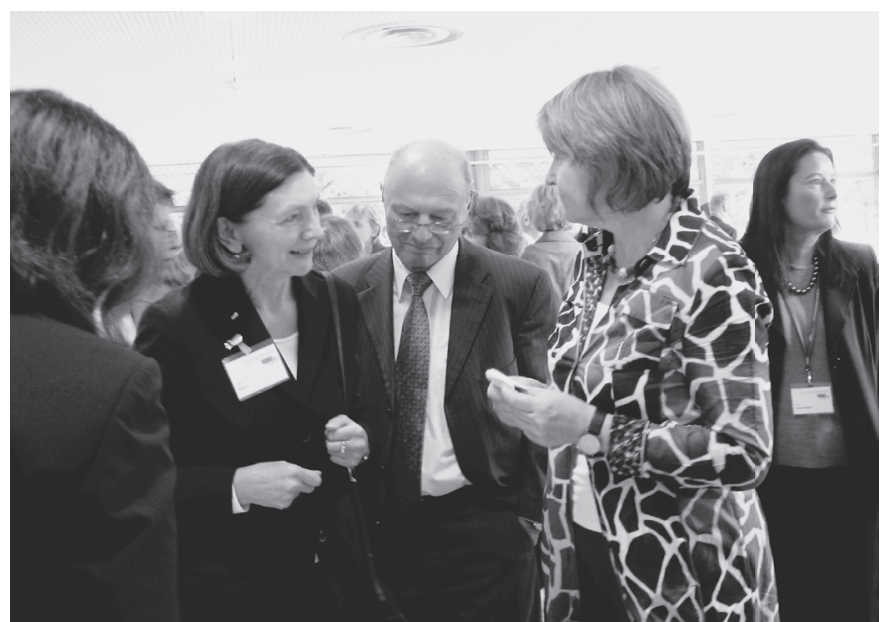

$\triangle$ Präsidentin Jutta Wagner (links) im Gespräch mit Ingrid Schmidt, Präsidentin des BAG (Foto: RM).

sind in der Schule erfolgreicher als Jungen - wenige von ihnen verlassen die Schule ohne oder nur mit einem Hauptschulabschluss.

Es ist mittlerweile auch unverkennbar, dass Frauen mit Migrationshintergrund in ihren Familien eine Schlüsselfunktion einnehmen - mehr noch als in der einheimischen Bevölkerung. Oftmals sind es gerade diese Frauen, die die Integration der nächsten Generation prägen. Sie sind Ansprechpartnerinnen für Kindergarten und Schule, für das soziale Umfeld und für Behörden; sie sind zuständig für die Gesundheit der Familie und die Freizeitgestaltung ihrer Kinder. Dabei ist zu berücksichtigen, dass Frauen mit Migrationshintergrund bei der Erziehung ihrer Kinder und bei der Gestaltung ihres Familienlebens oft vor besonderen Herausforderungen stehen. Sie streben ein gewisses Gleichgewicht zwischen der Bewahrung ihrer Herkunftskultur und den Anforderungen der so genannten Aufnahmegesellschaft an. Sie möchten einerseits die Traditionen und Werte ihres Heimatlandes bewahren, den Kontakt zu ihrer Herkunft nicht verlieren, Sitten und Gebräuche beibehalten, die sie ihrerseits von der eigenen Mutter gelernt haben. Andererseits ist es für sie wichtig, den Anschluss an die Gesellschaft, in der sie und ihre Familie leben, nicht zu verpassen; an eine Gesellschaft, deren Denken und Handeln nicht immer den Vorstellungen und Erfahrungen der Migrantinnen selbst entsprechen.

Dieses Gleichgewicht zu finden ist nicht immer leicht, oft geht es mit Konflikten einher; nicht immer gelingt dieser Prozess. Hier gilt es also, Unterstützung und Hilfestellung anzubieten. Diese ist speziell auf die Bedürfnisse, die Potenziale und die Stellung von Migrantinnen in der Familie auszurichten. Unsere zentralen integrationspolitischen Ziele müssen daher nach meiner Überzeugung darin bestehen,

- die Lebenssituation von Mädchen und Frauen mit Migrationshintergrund weiter zu verbessern;

- Migrantinnen über ihre grundlegenden Rechte gerade auch im häuslichen Umfeld zu informieren; 
- verstärkt Partizipationsmöglichkeiten am gesellschaftlichen, politischen und kulturellen Leben zu schaffen, auch durch Prozesse der interkulturellen Öffnung;

- Migrantinnen noch stärker in den Dialog über Erziehungsund Bildungsziele, Werte und Grundlagen unserer Gesellschaft einzubinden;

- klar zu machen, von welch zentraler Bedeutung das Thema Bildung und das Erlernen der deutschen Sprache für ihre Zukunft und die Zukunft ihrer Kinder ist.

Im Hinblick auf diese Zielrichtungen war es übrigens ein wichtiger Schritt, dass seit dem Inkrafttreten des Zuwanderungsgesetzes am 1. Januar 2005 im Rahmen des Integrationskurssystems spezielle Frauenkurse, zum Teil auch vorbereitende Seminarmaßnahmen, durchgeführt werden. Außerdem wurden entsprechend der Zielbestimmung des Nationalen Integrationsplans zum 1. Januar 2008 als weitere Themenschwerpunkte der Frauenkurse die Sprachentwicklung und der Spracherwerb der Kinder durch die Eltern sowie der Schutz vor häuslicher Gewalt aufgenommen.
Lassen Sie mich noch ein Thema ansprechen, mit dem sich die baden-württembergische Landesregierung seit Jahren intensiv beschäftigt: die Zwangsverheiratung von Mädchen und jungen Frauen. Es wird eine wichtige Aufgabe der neuen Bundesregierung sein, die Zwangsheirat als umfassenden Straftatbestand in das Strafgesetzbuch aufzunehmen, die zivilrechtliche Rechtsstellung von Opfern von Zwangsverheiratungen zu verbessern und im Aufenthaltsrecht ein verbessertes Rückkehrrecht in Fällen der Heiratsverschleppung zu verankern.

Meine sehr geehrten Damen und Herren: Das Potenzial von Frauen mit Migrationshintergrund liegt - trotz vieler positiver Entwicklungen - oft noch brach. Es gilt, die Schlüsselfunktion dieser Frauen verstärkt zu nutzen und Teilhabemöglichkeiten zu schaffen, um den Integrationsprozess weiter voranzubringen. In diesem Sinne wünsche ich Ihnen eine interessante und erfolgreiche Tagung und hoffe, dass Sie auch Gelegenheit finden, einen Eindruck von der Stadt Karlsruhe mit ihren Sehenswürdigkeiten und der typischen badischen Gastlichkeit zu bekommen!

Vielen Dank!

\section{Integration durch Verfassung Eröffnungsvortrag}

\section{Prof. Dr. Gertrude Lübbe-Wolff, Richterin des Bundesverfassungs- gerichts, Karlsruhe}

Welche Bedeutung hat die Verfassung für die Integration einer Gesellschaft oder politischen Gemeinschaft?

Die Frage, wie die Integration menschlicher Gesellschaften eigentlich funktioniert, wird gern gleichgesetzt mit der Frage: Was hält eine Gesellschaft zusammen? Diese Frage führt auf eine Merkwürdigkeit vieler Integrationsüberlegungen. Sie scheint vorauszusetzen, dass es ein Etwas gibt, das menschliche Gesellschaften zusammenhält, und schickt den Fragenden auf die Suche nach diesem Etwas. Vor einem Staat oder einer anderen Organisationsform menschlicher Gesellschaft steht man mit dieser Frage wie vor einer Sandkugel: Warum zerbröckelt sie nicht, warum zerrieselt sie nicht in lauter einzelne Sandkörner? Da muss doch irgendetwas für die Kohäsion Verantwortliches beigemischt sein! Dieses Etwas muss man finden, dann hat man die Erklärung für die Kugel, die man vor sich sieht, und damit auch das Backrezept für schöne, feste Sandkugeln.

\section{Die freiheitliche Verfassung - integrationsschwach?}

Die beliebteste Antwort auf die Frage, woraus er denn besteht, der Kitt, der die Gesellschaft zusammenhält, lautet, dass der Zusammenhalt nur durch irgendeine Art von Ähnlichkeit oder spezifischer Gemeinsamkeit, kurz: durch Homogenität, vermittelt und gesichert werden kann. Unterschiedlich sind dann aber wieder die Vorstellungen über die Art des benötigten Homogenitätskitts.

Über Homogenitätslehren, die auf Gleichheit der Rasse oder Ethnie setzten, ist hier kein Wort zu verlieren. Wo in der Staatslehre heute Homogenität als Voraussetzung gelingender politischer Gemeinschaft postuliert wird, kommt die Gemeinsamkeit der „Abstammung“, wenn überhaupt, dann nur noch als ein möglicher homogenitätsstiftender Faktor neben anderen vor, oder man zieht sich ganz auf eine irgendwo im Kulturellen (Gemeinsamkeit der Sprache, der Religion, der Geschichte usw.) angesiedelte Homogenität zurück. Oder, das ist die letzte Schwundstufe, auf irgendeine Form von Überzeugungs- oder Gefühlseinheit. Als der unverzichtbare Homogenitätskitt gilt dann beispielsweise ein Grundkonsens oder ein Wir-Gefühl. Auch die Idee vom Verfassungspatriotismus als einigendem Band gehört hierher.

Homogenitätslehren, die auf einer Art Gesinnungs- oder Gefühlseinheit beharren, haben eine gewisse Tendenz, in Resignation vor den widrigen Weltläuften zu enden: Man bemerkt das Faktum zunehmender gesellschaftlicher Differenzierung, also zunehmender Inhomogenität, erkennt womöglich auch noch, dass eine freiheitliche Verfassung gesellschaftlichen Differenzierungen und der damit einhergehenden Inhomogenität nicht entgegenwirkt, sondern sie im Gegenteil gerade ermöglicht und schützt, und sieht deshalb das haltende Gemeinsame der Erosion preisgegeben. 